\title{
Correlation between the Interface Width and the Adhesion Strength of Copper Films Deposited on Ion-Etched Carbon Steel Substrates by Varying the Bias Voltage
}

\author{
Abousoufiane Ouis ${ }^{1,2, *}$, Kamal Touileb ${ }^{1}$, Rachid Djoudjou ${ }^{1}$, \\ Abdulhakim Okleh ${ }^{1}$ \\ ${ }^{1}$ Mechanical Engineering Department, Prince Sattam bin Abdulaziz University, Al Kharj, Saudi Arabia \\ ${ }^{2}$ Laboratoire de Sciences des Surfaces et Interfaces en Mécanique, ISITEM, Université de Nantes, France
}

\begin{abstract}
The present study has been conducted in order to determine the influence of negative bias voltage applied to substrate on adhesion of copper films deposited on carbon steel substrates. The adhesion strength has been evaluated by the scratch test. Coatings were deposited by a DC magnetron sputtering system. The substrates were firstly mechanically polished and then ion-etched by argon ions prior to deposition. Adhesion was found to increase with the bias voltage. The critical load had a value of $9.5 \mathrm{~g}$ for an unbiased substrate and reached $18.5 \mathrm{~g}$ for a bias voltage of $-600 \mathrm{~V}$. Equally important, the interface width, measured using Auger electron spectroscopy, increased as a function of the bias voltage. The width of the interface is related to the time of ion milling in the Auger spectrometer. The size of this width is obtained from the Auger elemental depth profiles through measuring the depth of the interface coating/substrate. The width had a value of 335 min with a bias of $-600 \mathrm{~V}$ whereas it didn't exceed $180 \mathrm{~min}$ when the substrate was unbiased. Therefore, the effect of the bias voltage was to expand the interface because of the diffusion phenomenon and physical mixing of materials at the interface. Moreover, the critical load increased with the increase of the interface width.
\end{abstract}

Keywords: DC sputtering, Ar ion bombardment, Bias voltage, Adhesion, Auger electron spectroscopy.

\section{Introduction}

In the industrial production of coatings, one of the important problem to surmount is the maximization of coating to substrate adhesion strength. To do this, many processes are used such as ion bombardment of the substrate surface, heating of specimens, deposition of an intermediate layer, application of a substrate bias voltage and others. Application of a substrate bias voltage on the substrate during the film growth process has been identified as a very promising technology for producing well adherent coatings. It can greatly modify the mechanical properties particularly the adhesion, tribological properties, morphology, crystallographic composition of a wide variety of film-substrate. Seeman published a paper on the knowledge and understanding of the biasing technique [1] that had a two-fold purpose: First, to provide persons in science and industry interested in thin film technology with a basic understanding of the theory, mechanisms and techniques of bias sputtering; and second, to point out the versatility of this technique by illustrating its use in practical applications. It is noted that the theory and mechanisms of the biasing action are very similar to those seen in the basic ion sputtering concept. For instance, in a study of TiAlLaN films deposited on WC-Co and on silicon substrates [2], Hao Du et al. found that by increasing the bias voltage from -30 to $-70 \mathrm{~V}$, titanium concentration increased from 34.51 to $38.05 \%$ and nitrogen atomic content remained almost constant. However, aluminum and lanthanum concentrations decreased from 14.39 to $12.37 \%$ and from 1.51 to $0.73 \%$ respectively. This variation has been explained by a phenomenon of ionization and the backscattering of deposited elements. The grains of the films changed from equiaxial to columnar structure and the grain size changed from 36.44 to 36.33 $\mathrm{nm}$ due to the alteration of film surface energy under the bias voltages. Moreover, the mechanical properties of the films notably changed. For instance, the increase in the critical load of the adhesion scratch test is from 75 to $84 \mathrm{~N}$, for the hardness is from 29.48 to $38.27 \mathrm{GPa}$ and for the elastic modulus is from 449.15 to $667.35 \mathrm{GPa}$. Deviaet al. reported their results [3] related to TiAIN coatings deposited on AISI O 1 tool steel using a triode magnetron sputtering system by varying the bias voltage from -40 to $-150 \mathrm{~V}$. The titanium content decreased from 32 to 30 at.\% and the aluminum content increased slightly from 25 to 28 at.\%, while the nitrogen content was found to remain constant. The transformation of the crystallographic structure of the film from preferred $\{1$ $11\}$ to random $\left\{\begin{array}{lll}2 & 0 & 0\end{array}\right\}$ oriented with increasing the bias voltage was due to the reduced average ion energy. Moreover, the grain size of the film increased from 51.3 to $96.3 \mathrm{~nm}$ leading to a decreasing of the hardness from 32 to $19 \mathrm{GPa}$. The film thickness showed an inversely proportional relationship with the bias voltage as it decreased from 1.875 to $1.295 \mu \mathrm{m}$. Although this technique tends to produce denser films. In other work of $\mathrm{Cr}_{2} \mathrm{O}_{3}$ films deposited by arc ion plating on AISI 304 stainless steel substrates [4], Wang et al. found that with increasing the bias voltage from $0 \mathrm{~V}$, the hardness increased gradually from $27.6 \mathrm{GPa}$ to reach the highest value 
of about $32.2 \mathrm{GPa}$ at $-100 \mathrm{~V}$, and then sharply decreased to $25.4 \mathrm{GPa}$ at $-250 \mathrm{~V}$. Moreover, the critical load of adhesion scratch test first increased from about $29 \mathrm{~N}$ to the maximum of $46.7 \mathrm{~N}$ when the bias changed from 0 to $-100 \mathrm{~V}$ and then decreased gradually to about $32 \mathrm{~N}$ at a bias voltage of $-250 \mathrm{~V}$. The wear testing also revealed that the bias voltage modified the friction coefficient of film. The origin of these phenomena was attributed to the microstructure evolution and variation of defect density in the films induced by the negative bias voltage increase. Kovacet al. investigated the influence of substrate bias voltage on the properties of sputtered aluminum-scandium thin sheets deposited on steel sheets by a DC magnetron sputtering unit [5]. The results showed that the bias voltages caused a decrease in the deposition rate due to the resputtering of the surface atoms. When the negative bias voltage varied from 0 to $250 \mathrm{~V}$, the film morphology changed from a fine to an irregular crystalline structure. Moreover, the micro-hardness of the sheets showed a monotonically increase from about $150 \mathrm{HV}$ for an unbiased substrate to about $635 \mathrm{HV}$ for a bias voltage of $-250 \mathrm{~V}$. In the same range of bias voltage, the increase of the applied bias resulted in an augmentation of the free surface roughness. This rise was relatively limited for small bias voltage but increased dramatically for values above $150 \mathrm{~V}$. On other hand, inert gas ions or other ion species with high, medium, and low energies have been used, before or during film deposition process, to induce adhesion enhancement between deposited films and substrates. The main effects of ions bombardment by argon ions could be summarized by the oxides layer removal and a roughness creation on the substrate surface, and the substrate temperature increase. Donnelly and Kornblit [6] have reviewed the field of plasma etching in a long review article entitled "Plasma etching: Yesterday, today, and tomorrow". They presented its history, different categories, equipments, evolution, mechanisms and applications. Nevertheless, the choice of the technique for evaluating the adhesion of thin films on their substrates remains a major issue. Many techniques of measuring the adhesion have been developed and evaluated [7]. The well established "scratch test" using a hemispherical diamond stylus, loaded tangentially to the plane of the surface offers a high degree of usability and reproducibility [8].

In this work, the influence of a negative bias voltage applied to the substrate on the adhesion strength coating-substrate was explored. The films of pure copper were fabricated by a DC magnetron sputtering system. The substrates were made of E24 carbon steel. Before the deposition process, the substrate surfaces were treated by argon ions etching. Consequently, the oxides layer at the substrate surface was removed and, an increase of temperature and roughness of the substrate were generated. The bias voltage that was applied during all the film growth was varied from 0 to $-600 \mathrm{~V}$. The attention was focused on the correlation between the interface film/substrate width and the adhesion strength. The adhesion scratch test was used to evaluate the adhesion. Whereas, the physicochemical nature of the interface and the inter-diffusion phenomenon between copper film and steel substrate were assessed through Auger electron spectroscopy depth profiles. The obtained results revealed that the interface width was an increasing function of the bias voltage. Moreover, critical load of the scratch test increased with the increase of the interface width. These observations suggested that the bias voltage had effects on one hand of diffusion and mixing of the materials at the interface, and on other hand of increasing of the substrate temperature. Consequently, the latter effects contributed positively to the adhesion enhancement of the film to the substrate.

\subsection{Substrate preparation}

\section{Experimental Details}

Common E24 carbon steel was used as substrate in this study. Chemical composition of this material is listed in Table 1. The substrates were cut into pieces with sizes of $15 \times 15 \times 2 \mathrm{~mm}$ and their surface preparation involved a sequential hand grinding with emery abrasive paper grades 600, 800 and 1200. Subsequently, a polishing with diamond paste (particle sizes 6.0 and $1.0 \square \mathrm{m}$ ) was performed in order to obtain a mirrorlike finished substrate. After a cleaning procedure in ultrasonic baths of acetone and methanol, successively for about 15 minutes for each, the substrate was rinsed in distilled water and finally dried under a stream of heated air. Then, the substrate was immediately introduced in the deposition chamber.

Table 1.Chemical composition of E24 carbon steel

\begin{tabular}{|l|l|}
\hline Chemical elements \%, by mass & \\
\hline $\mathrm{C}(\max )$ & 0.17 \\
\hline $\mathrm{P}(\max )$ & 0.045 \\
\hline $\mathrm{S}(\max )$ & 0.045 \\
\hline $\mathrm{Mn}(\max )$ & 1.40 \\
\hline $\mathrm{Fe}$ & balance \\
\hline
\end{tabular}

\subsection{Coating deposition}

The pure copper films were deposited by a DC magnetron sputtering system. When the sample was introduced in the vacuum chamber, the chamber was first evacuated by a rotary vane pump to $\approx 1 \mathrm{~Pa}$ and then pumped down by a diffusion pump to reach a base pressure of $(5-7) \times 10^{-4} \mathrm{~Pa}$. The argon gas was leaked to the 
chamber through a needle valve. The used deposition parameters are pressure of $1 \mathrm{~Pa}$, cathodic voltage of $560 \mathrm{~V}$ and current intensity of 1.8 A corresponding of a power density of $11.6 \mathrm{~W} . \mathrm{cm}^{-2}$ and during all the processes, a bias voltage was applied to the substrate by using an RF power supply. So, three series of specimens were produced and analyzed with negative bias voltages of 0,300 and $600 \mathrm{~V}$. Nevertheless, all the samples received before deposition process an in situ ion bombardment etching treatment with argon ions. The experimental conditions of ion bombardment etching that were obtained in a previous study [9] represent the optimal conditions giving the higher critical load in the adhesion scratch test namely argon pressure of $10 \mathrm{~Pa}$, cathodic voltage of $600 \mathrm{~V}$ and ion etching time of $10 \mathrm{~min}$. When the ion etching was performed, the pressure in the vacuum chamber was adjusted to the desired pressure, the bias voltage was immediately applied to the substrate and the film deposition process started. However, the specimens were kept for about 3 min between the end of the ion etching and the beginning of the deposition. As the application of a bias voltage causes an ion bombardment of the film surface which causes a resputtering of the surface atoms and thus decreases the deposition rate, the sputtering time was varied so that the film thickness was kept in a reasonable range of 200 $\mathrm{nm}$. The detailed parameters used for the fabrication of these samples are listed in Table 2.

Table 2. Detailed parameters for pure copper films fabricated by DC magnetron sputtering technique

\begin{tabular}{|l|l|}
\hline Parameters & Value \\
\hline Base pressure $(\mathrm{Pa})$ & $(5-7) \times 10^{-4}$ \\
\hline Working pressure $(\mathrm{Pa})$ & 1 \\
\hline Deposition DC cathodic voltage $(\mathrm{V})$ & 560 \\
\hline Deposition current intensity $(\mathrm{A})$ & 1.8 \\
\hline Deposition energy density $\left(\mathrm{W} . \mathrm{cm}^{-2}\right)$ & 11.6 \\
\hline Ar ion bombardement pressure $(\mathrm{Pa})$ & 10 \\
\hline Ar ion bombardement voltage $(\mathrm{V})$ & 600 \\
\hline Ar ion bombardement time $(\mathrm{min})$ & 10 \\
\hline RF bias voltage $(\mathrm{V})$ & $0,-300,-600$ \\
\hline Film thickness $(\mathrm{nm})$ & 200 \\
\hline
\end{tabular}

\subsection{Measurement of the critical load}

Adhesion of the coating on the substrates was assessed by the adhesion scratch test. The principle of the test consists of moving a loaded stylus with small weights and visually analyzing the scratch track. The stylus is made of diamond with a tip radius of $17 \square \mathrm{m}$ and mounted on a beam. To perform a scratching, first of all the sample was positioned and fixed in a substrate-holder that was an $x-y$ table. A DC motor allowed the movement of the table in the $x$-direction and, for a given value of the load, a scratch was obtained. In our experiments, we adopted scratches of about $5 \mathrm{~mm}$ long and a constant scratch speed of $3.7 \mathrm{~mm} \cdot \mathrm{min}^{-1}$. On the same sample, a series of parallel scratches was manually performed by moving the table in $y$-direction using of a micrometer screw. From one series of scratches to the other, the load was increased by a discrete value of the load until the coating/substrate adhesion failure occurred. The shapes of the scratch tracks were analyzed by an optical microscope and micrographs were taken. For a given load, a series of at least three scratches were realized and each channel was separately examined by optical inspection. Thus, the adhesion failure was estimated by a mean critical load.

\subsection{Adhesion measurement}

\section{Results and Discussion}

The surface treatment by argon ions bombardment prior to deposition process that has major effects of removing oxides layer, creating a roughness on the substrate surface and elevating the substrate temperature plays an important role in the adhesion improvement [10-13]. For instance, Lim et al. [14] studied the effects of the surface roughness of $96 \%$ pure $\mathrm{Al}_{2} \mathrm{O}_{3}$ substrates on the adhesion of $99.99 \%$ pure copper films. Trapezium rule of calculation in 2D model for estimated total surface area has been introduced. The various roughness grades have been analyzed using Atomic Force Microscopy and the adhesion strength has been evaluated with pull strength measurements. With rougher surface, the adhesive strength is higher due to the larger contact area at the interface. This effect can account for an adhesive strength increment of more than 50\%. Additionally, Lahmar et al. [15] who investigated the adhesion of the similar previous coating-substrate by the scratch test have drawn almost similar conclusions. The substrates were ion etched before deposition by Ar ions. The increase in the scratch test critical load was caused by a better adhesion of the coating to its substrate resulting from a widening of the interface region. On other hand, the argon ion etching eliminated the contamination (oxygen and carbon) and created vacancies and microcavities on the surface substrate, and produced an important contact surface coating-substrate allowing a good anchoring of the coating on its substrate. In our work, as all samples received the same surface treatment by argon ions etching prior to deposition. This treatment has no effect on the variation of the critical load as a function of the bias voltage. Moreover, the bias voltage has effects, among others, of physical mixing of materials in the interfacial domain, increasing of the diffusion and improving the deposited film quality (greater densification). Hence, these effects of the ion etching 
and the bias voltage, when combined, give an enhancement of the adherence greater than this one obtained if only one of the effect is applied. Table 3 shows the variation of the mean critical load in the adhesion scratch test as a function of the substrate negative bias voltage. The critical load increases as a function of the bias voltage. It has a value of about $9.5 \mathrm{~g}$ for an unbiased substrate and reaches a value of about $18.5 \mathrm{~g}$ for a bias voltage of $-600 \mathrm{~V}$. Hence, the amplification factor is of about 2. The literature review reveals that the effects of the bias voltage variation on the adhesion of films to their substrates can be mainly attributed to some factors such as hardness, structure, chemical composition of the coating and internal stress in the coating generated by the bias voltage as well as to the deposition parameters. Thus, prior to deposition of a-C:H films, Wazumiet al. [16] sputter-cleaned the silicon wafer by Ar plasma applying a negative DC voltage of $2.0 \mathrm{kV}$ for $30 \mathrm{~min}$. Afterward, films prepared at different bias voltages presented an improvement of the adhesion due to produced low compressive stress. Other results have been reported by Shi et al. [17] when investigating the adhesion properties of the nano-scaled CrTiAlN multilayer coatings deposited on $\mathrm{Mg}$ alloy substrates by unbalanced magnetron sputtering. The results of the scratch test with a progressive load indicated that the adhesion force of the coating was strongly affected by the negative substrate bias voltage. They revealed that the critical load was only about $3.75 \mathrm{~N}$ at $-40 \mathrm{~V}$ and reached $10 \mathrm{~N}$ at $-50 \mathrm{~V}$. However, the critical load value decreased with the increase in bias voltage and the reason for this behavior could be cited to the generation of excess compressive stresses due to the bombardment by high energy ions, and the consequent detrimental of the coating adhesion. Hence, the authors indicated that the adhesion property of the coatings is subject essentially to the chemical composition and deposition parameters. Comparable results have been reported by Valeriniet al. [18] in the case of $\mathrm{ZrN}$ films sputtered on tungsten carbide substrates. Beside fracture energy, microhardness and internal stress, Müller and Fromm investigated the adhesion strength of TiN coatings on HSS substrates as a function of the negative bias voltage [10]. The critical load, increased from 9 to $17 \mathrm{~N}$ with increasing bias voltage from 0 to $-120 \mathrm{~V}$. The hardness values were constant from 0 to $-20 \mathrm{~V}$. Between -20 and $-40 \mathrm{~V}$ they steeply increased from 1910 to $2600 \mathrm{HV}$ but for higher bias voltage they did not change any more. The different behavior of the scratch test and the hardness values were caused by the change of the structure of the TiN coating due to application of a bias voltage. Argon ions accelerated in the field of the bias voltage hit the substrate surface and enhanced the mobility of the surface atoms. This had the consequence that the open, porous, columnar coating structure was converted to a denser film. Nevertheless, the change in the coating structure increased the values by a factor of two in a similar way as the hardness values. The authors concluded that the critical load was obviously more affected by the change of the coating hardness than by the change of the adhesion strength.

Table 3. Critical load of adhesion scratch test as a function of the substrate bias voltage. Data of the copper films prepared using negative bias voltage from Ouiset al. [9]

\begin{tabular}{|r|r|r|r|}
\hline Substrate bias voltage $(-\mathrm{V})$ & 0 & 300 & 600 \\
\hline Mean critical load $(\mathrm{g}) \pm 0.5 \mathrm{~g}$ & 9.5 & 12.0 & 18.5 \\
\hline
\end{tabular}

The results of the adhesion scratch test were analyzed by an optical microscope. Figure 1shows micrographs of the scratch tracks obtained for substrates argon ion etched before deposition and coated with copper applying a $-300 \mathrm{~V}$ bias voltages during deposition process. Under these conditions for such specimens, the mean critical load is about $12.0 \mathrm{~g}$. Figure 1(a) is related to an applied load of $11 \mathrm{~g}$. The scratch channel has a uniform appearance revealing that the coating remains in a good adherence to the substrate. So, this load is less than the critical load. In Figure 1(b), the load is $12 \mathrm{~g}$ and small film loosening starts to appear along the scratch track edges. Hence, this load can be approved as the critical load. Whereas, applying a load of $15 \mathrm{~g}$, scratch track shows clearly that the film is totally removed under the stylus as shown in Figure 1(c). Consequently, the applied load is much greater than the critical load and it is an evidence that the adhesion loss is complete.

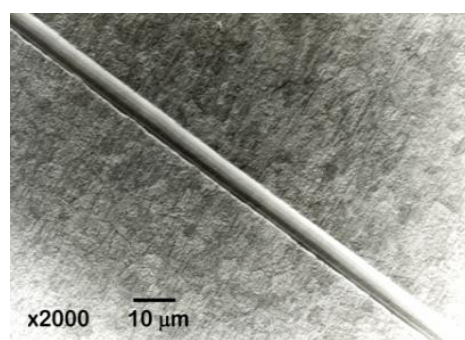

(a)

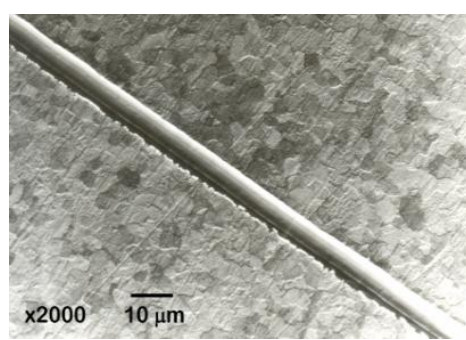

(b)

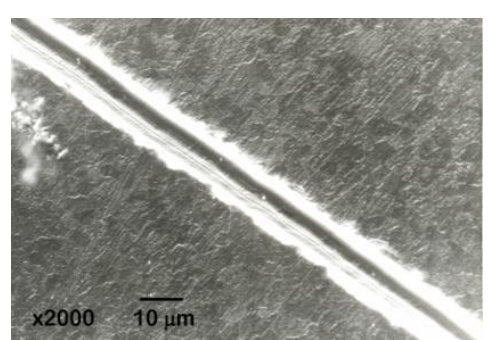

(c)

Figure 1. Optical microscopy micrographsof scratch track of the scratch test of copper films deposited on ion etched substrates with an applying bias voltage of $300 \mathrm{~V}$.The mean critical load was $\approx 12 \mathrm{~g}$. The applied stylus load was (a) $11 \mathrm{~g}$, (b) $12 \mathrm{~g}$ and (c) $15 \mathrm{~g}$. 


\subsection{Auger electron spectroscopy}

Auger electron spectroscopy (AES) was used to determine the chemical elements present in the coating, in the interface coating/substrate and in the substrate as well as to measure the interface width. An elemental depth profile was derived from a series of Auger spectra taken at different ion milling time by plotting the peak-to-peak heights of the principal Auger lines as a function of ion milling time. According to the obtained AES results, the main chemical elements detected are copper, iron, oxygen and/or carbon. Figure 2 is related to samples ion bombardment etched before depositions under the optimal conditions of ion etching before deposition. Figure 2(a) corresponds to an unbiased substrate. Figures 2(b)and 2(c) correspond to substrates where negative bias voltages of 300 and $600 \mathrm{~V}$ are respectively applied during deposition. For all substrates prepared with or without bias voltage, a superficial contamination has been observed at the coating surface. This contamination comes from the formation of a superficial layer during the transfer of the sample in the atmosphere from the deposition chamber to the Auger spectrometer. This generated layer is removed by the first ion millings in the AES spectrometer. Moreover, an obtained and expected result is that for all the samples, the oxygen is absent in the interface. When performing mechanical polishing of the substrates before their introduction to the deposition chamber, an amorphous layer of oxides is generated on the substrate surface. This layer is removed by the ion bombardment etching. Hence, the interface presents no contamination. In the case of an unbiased sample (Figure 2(a)), the oxygen is completely absent however traces of carbon are present in the interface and in the substrate. This presence is probably due to the carbon contained in the substrate composition. In that case, it is important to remember that the samples were prepared from commercial quality steel with a not perfectly uniform distribution of the constituent elements.

The AES depth profiles allowed us to measure the width of the interface for different applied bias voltages. The interface begins to form when the iron starts to increase and the copper starts to decrease. Thus, the interface is considered as finished when the iron remains constant and the copper disappears. Based on the aforementioned considerations, the interface width is measured in minutes, corresponding of the ion milling time in the AES spectrometer. As far as the effects of the bias voltage on the interface width and then on the adhesion coating/substrate are considered, Figure 3 and 4 are plotted. Figure 3 presents the results of the variation of the interface width as a function of the bias voltage. It is clear that when the bias increases, the interface width increases. When the bias had values of 0,300 and $600 \mathrm{~V}$, the corresponding interface widths were 180, 270 and 335 min respectively. It is evident that the interface is significantly more wide and more diffuse for a biased substrate than for an unbiased substrate where the interface is more abrupt. Therefore, application of a bias voltage on the substrate has a positive effect in the enlargement of the interface due to diffusion phenomenon and intermixing of materials at the interface.

According to the results of the adhesion scratch test (cf. Table 3), Figure 4 presents the relationship between the mean critical load and the interface width. The mean critical load is an increasing function of the interface width. When the interface width has a value of $180 \mathrm{~min}$ le critical load is $9.5 \mathrm{~g}$ for an unbiased substrate. However, when the interface width increases to a value of $335 \mathrm{~min}$ the critical load becomes $18.5 \mathrm{~g}$ for an applied bias voltage of $600 \mathrm{~V}$. The critical load is significantly influenced by the application of a bias voltage. Hence, the latter parameter has an important contribution in the adhesion enhancement of the coating to its substrate.

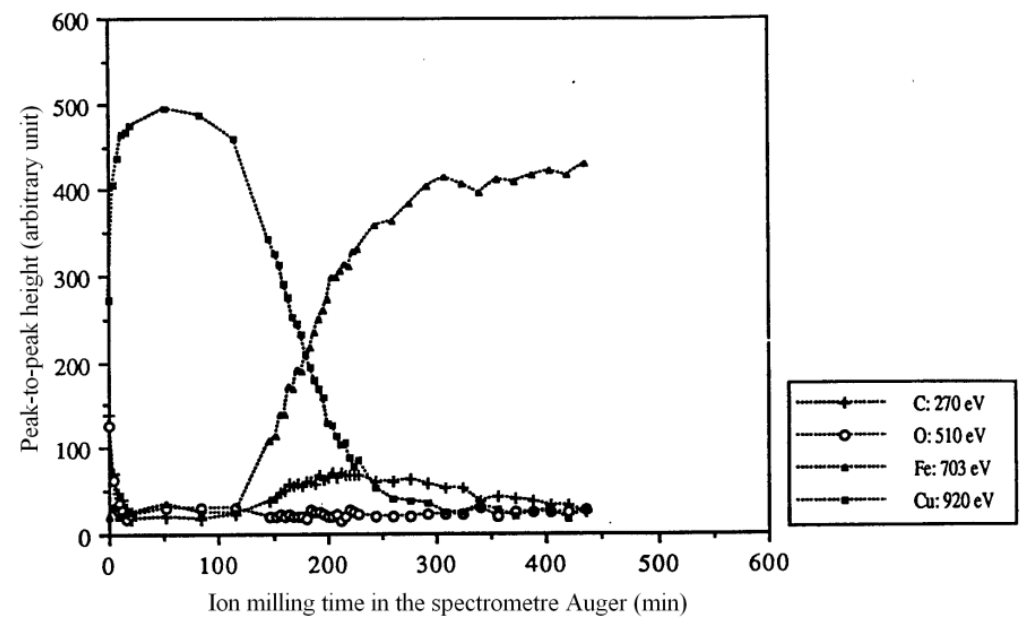

(a) 


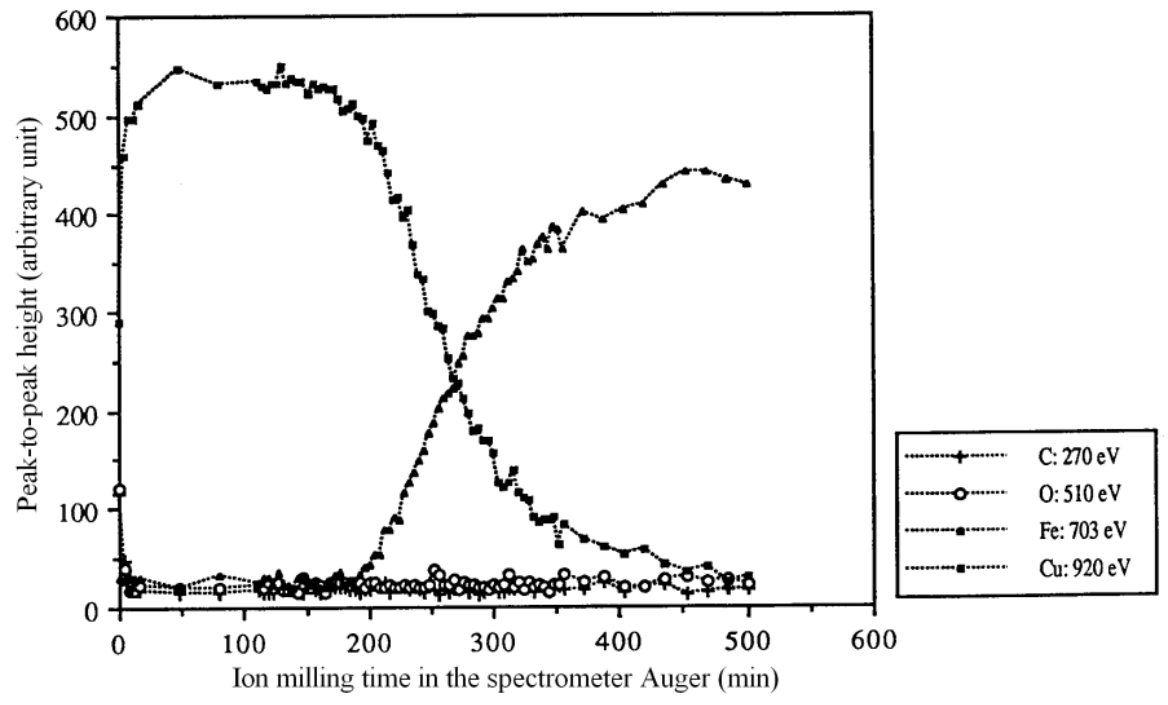

(b)

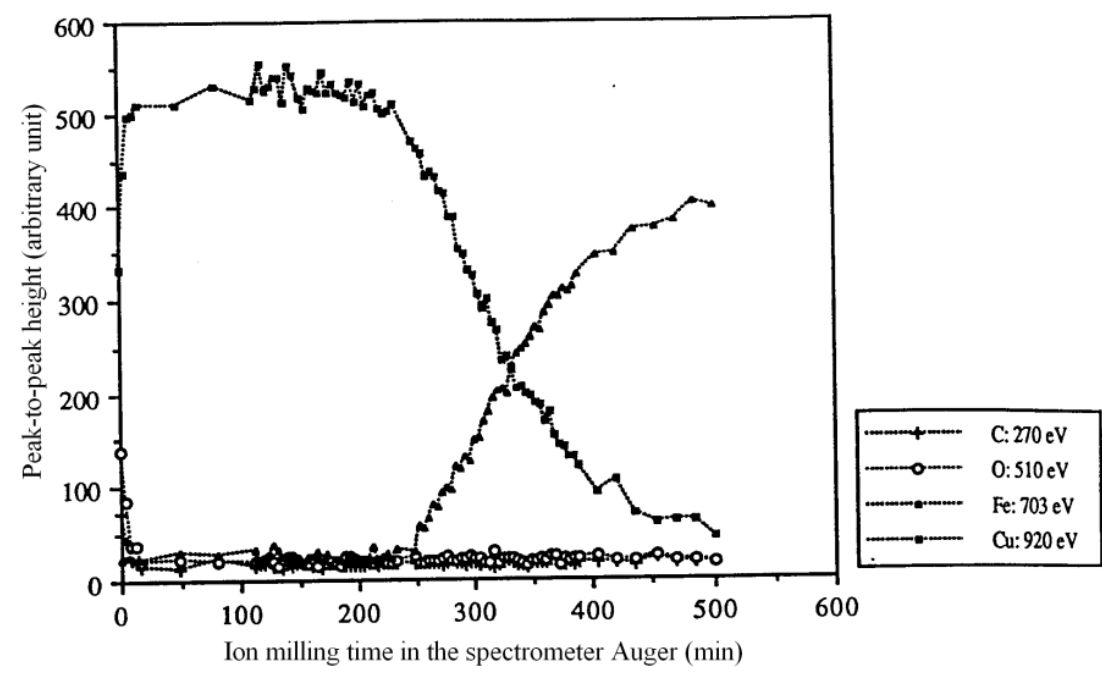

(c)

Figure 2. Auger depth profiles obtained for copper films onto E24 carbon steel substrate. Before deposition, the substrate was ion etched at $600 \mathrm{~V}$ and $10 \mathrm{~Pa}$ and $10 \mathrm{~min}$ and, during deposition, a substrate bias voltage was applied of (a) $0 \mathrm{~V}$, (b) $-300 \mathrm{~V}$ and (c) $-600 \mathrm{~V}$.

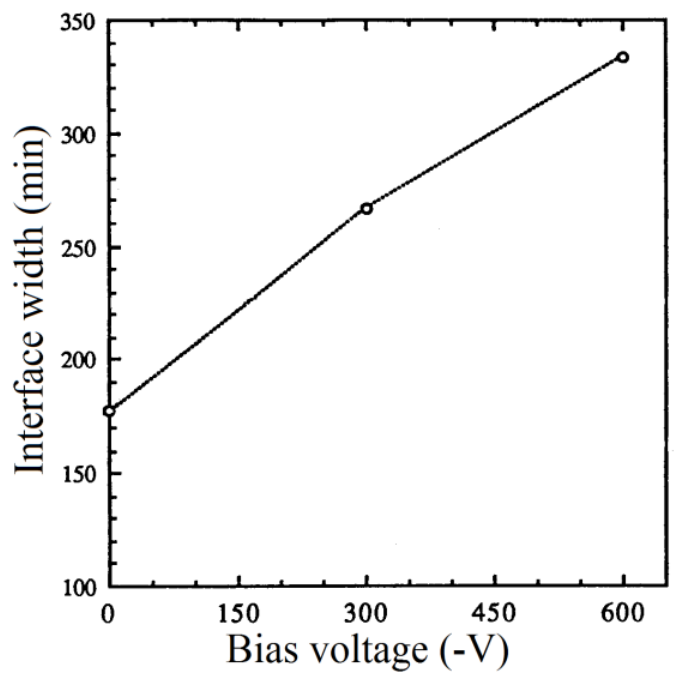

Figure 3. Variation of the interface width measured in the Auger spectra as a function of the bias voltage. 


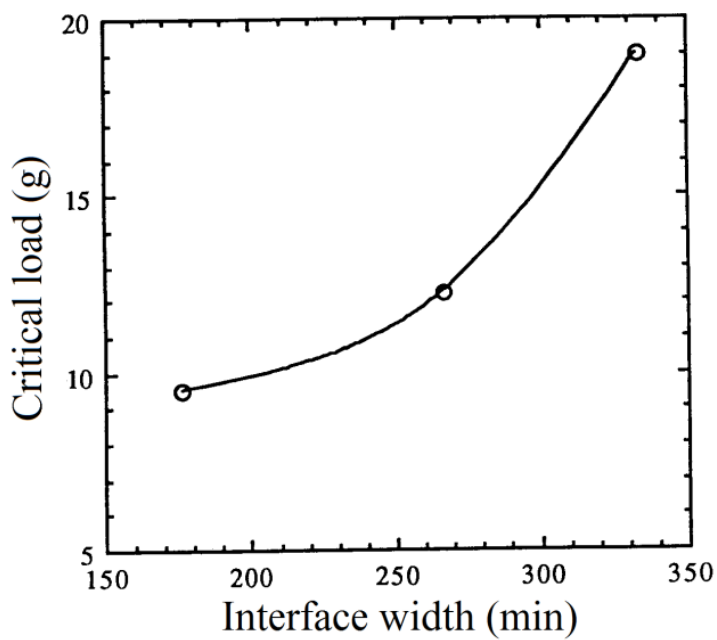

Figure 4. Variation of the critical load as a function of the interface width.

Our results are in good agreement with those obtained by Tang et al.[19] when studying the adhesion strength of TiN films synthesized on GCr15-bearing steel using plasma immersion ion implantation and deposition. Prior to the deposition process, the samples were treated by Ar+ sputtering. They found that the critical load in the adhesion scratch test increased from 1.80 to $2.89 \mathrm{~N}$ when the sputtering time varied from 0 to $30 \mathrm{~min}$ respectively. In addition, when the bias voltage was increased from -10 to $-30 \mathrm{kV}$, the adhesion strength raised from 2.37 to $14.6 \mathrm{~N}$ respectively. As the interface width was estimated by the nitrogen concentration in the interface induced by the implantation voltage, the authors observed that high implantation voltage resulted in a high implantation depth. For example, the critical load increased from 1.84 to $10.37 \mathrm{~N}$ when the nitrogen implantation varied from 0 to $-8 \mathrm{kV}$. Consequently, when the nitrogen concentration in the interface increased, the interface width increased and the adhesion strength increased also. Regarding the materials intermixing effects at the interface, Cheng et al. [20] investigated by a scratch test the adhesion enhancement of $(\mathrm{Cu}, \mathrm{Nb}, \mathrm{Ti})$ films deposited by evaporation on $\mathrm{SiO}_{2}$ at room temperature and at $300^{\circ} \mathrm{C}$ and using $\mathrm{Ar}$ ions bombardment to various doses. The experimental results showed that the adhesion strength increased after bombardment at a threshold dose and then saturated at higher doses in all pairs. The possible mechanism involved in this phenomenon has been mainly attributed to the nuclear energy loss. The nuclear energy loss during ion beam processing induces many defects in the solids such as vacancies, interstitials and their clusters, thermal spikes, etc... inducing a layer of intermixing atoms at the interface coating/substrate. However in our study, a wide range of factors, such microhardness and internal stress in the coating as well as film crystallography will affect the values of the critical load when a bias voltage is used during the deposition process. Further investigation is needed to explore the relationship between these factors and the scratch adhesion.

\section{Conclusions}

Pure copper films were fabricated by a DC magnetron sputtering unit. Prior to deposition, the carbon steel substrates were argon ion etched. Different negative bias voltages were applied on the substrates during the deposition process. Critical load in the adhesion scratch test has been measured. Effects of the negative substrate bias voltage on the interface width and on the critical load were studied. Auger electron spectroscopy (AES) has been used to analyze the coating, the interface coating/substrate and the substrate. Results of this study allow to draw the following conclusions:

1. During the transfer of the sample in the atmosphere from the deposition chamber to the Auger spectrometer, the coating surface presents a contamination characterized by a layer of oxides. This layer is removed by the first ion millings in the AES spectrometer.

2. The mechanical polishing of the substrates generates at the surface substrate an amorphous layer of oxides and carbon. The optimal conditions of the ion bombardment are opted for etching the substrates before the deposition process (argon pressure: $10 \mathrm{~Pa}$, cathodic voltage: $600 \mathrm{~V}$, ion etching time: $10 \mathrm{~min}$ ). This ion etching eliminates the amorphous layer and creates vacancies and microcavities that produces an important contact surface coating-substrate allowing a good adhesion of the coating to the substrate.

3. The critical load is an increasing function of the bias voltage. It changes from a value of $\approx 9.5 \mathrm{~g}$ without bias to $\approx 18.5 \mathrm{~g}$ at $600 \mathrm{~V}$ bias voltage. This variation could be explained by the effect of the increasing of substrate temperature and by the mechanical anchoring of the coating to its substrate on the one hand (due to ion etching) and by the diffusion and the materials mixing in the interfacial domain (due to the substrate bias voltage) on the other hand. 
4. The bias voltage has a noteworthy effect on the interface width enlargement. The interface width has a value of $335 \mathrm{~min}$ for a bias of $600 \mathrm{~V}$ whereas it has only $180 \mathrm{~min}$ for an unbiased substrate. Thus, the interface is wider (diffuse) for a biased substrate than for an unbiased one (abrupt). This phenomenon is mainly due to the diffusion and intermixing of the materials in the interface. The temperature generated by the bias voltage has also a contribution.

5. The critical load is an increasing function of the interface width. It reaches $\approx 18.5 \mathrm{~g}$ for an interface width of $335 \mathrm{~min}$ whereas it is $\approx 9.5 \mathrm{~g}$ for $180 \mathrm{~min}$. The amplification factor is about 2 . As a result, a strong relationship exists between the interface width and the adhesion; the higher interface width, the better adhesion.

\section{Acknowledgements}

The authors express their gratefulness to Dr. D. Roptin and Mr. J. P. Roche (EcoleCentrale de NantesUniversity of Nantes-France) for the Auger electron spectroscopy analyses.

\section{References}

[1] J.M. Seeman, Bias sputtering: its techniques and applications, Vacuum, 17(3), 1967, 129-137.

[2] H. Du, J. Xiong, H. Zhao, Y. Wu, W. Wan, L. Wang, Structure and properties of TiAlLaN films deposited at various bias voltages, App. Surf. Sci.,292, 2014, 688-694.

[3] D.M. Devia, E. Restrepo-Parra, P. J. Arango, A. P. Tschiptschin, J. M. Velez, TiAlN coatings deposited by triode magnetron sputtering varying the bias voltage, App. Sur. Sci., 257(14), 2011, 6181-6185.

[4] T-G. Wang, D. Jeong, Y. Liu, Q. Wang, S. Iyengar, S. Melin, K.H. Kim, Study on nanocrystalline Cr2O3 films deposited by arc ion plating: II. Mechanical and tribological properties, Surf. Coat. Technol., 206, 2012, 2638-2644.

[5] J. Kovac, H-R Stock, H-W Zoch, Influence of substrate bias voltage on the properties of sputtered aluminum-scandium thin sheets, J. Surf. Eng. Mat. Adv. Tech., 2, 2012, 115-119.

[6] V.M. Donnelly, A. Kornblit, Plasma etching: Yesterday, today, and tomorrow, J. Vac. Sci. Technol., A 31, 050825, 2013,doi: 10.1116/1.4819316 (48 pages).

[7] J. Valli, A review of adhesion test methods for thin hard coatings, J. Vac. Sci. Technol., A 4, 1986, $3007-3014$.

[8] T.R. Hull, J.S. Colligon, A.E. Hill, Measurement of thin film adhesion, Vacuum, 37(3-4), 1987, 327-330.

[9] A. Ouis, M. Cailler, Effects of substrate bias voltage on adhesion of DC magnetron-sputtered copper films on E24 carbon steel: investigations by Auger electron spectroscopy, J. Adhesion Sci. Technol., 27(21), 2013, 2367-2386.

[10] D. Müller, E. Fromm, Mechanical properties and adhesion strength of TiN and Al coatings on HSS, steel, aluminium and copper characterized by four testing Methods, Thin Solid Films, 270, 1995, 411-416.

[11] M. Cailler, A. Ouis, P.J. Schultz, P.J. Simpson, The adhesion strength of copper thin-films to E24 carbon steel: effects of substrate surface ion bombardment etching, J. Adhesion Sci. Technol., 7(2), 1993, 141-157.

[12] K-D. Bouzakis, S. Makrimallakis, G. Katirtzoglou, G. Skordaris, S. Gerardis, E. Bouzakis, T. Leyendecker, S. Bolz, W. Koelker, Adaption of graded $\mathrm{Cr} / \mathrm{CrN}$-interlayer thickness to cemented carbide substrates' roughness for improving the adhesion of HPPMS PVD films and the cutting performance, Surf. Coat. Technol., 205(5), 2010, 1564-1570.

[13] F.H. Sun, Z.M. Zhang, M. Chen, H.S. Shen, Improvement of adhesive strength and surface roughness of diamond films on Cocemented tungsten carbide tools, Diamond Relat. Mater., 12(3-7), 2003, 711-718.

[14] J.D. Lim, Y.S.Y. Susan, R.M.W. Daniel, K.C. Leong, C.C. Wong, Surface roughness effect on copper-alumina adhesion, Microelectron. Reliab.,53(9-11), 2013, 1548-1552.

[15] A. Lahmar, N. Hmina, Y. Scudeller, J.P. Bardon, Correlation between the adhesion and the thermal contact resistance: effects of substrate surface ion bombardment etching, Thin Solid Films,325(1-2), 1998, 156-162.

[16] K. Wazumi, Y. Koga, A. Tanaka, Tribological properties of a-C:H films on Si substrate prepared by plasma CVD in pulse-biased process, Diamond Relat. Mater.,12, 2003,1018-1023.

[17] Y. Shi, S. Long, S. Yang, F. Pan, Deposition of nano-scaled CrTiAlN multilayer coatings with different negative bias voltage on Mg alloy by unbalanced magnetron sputtering, Vacuum, 84, 2010, 962-968.

[18] D. Valerini, M.A. Signore, L. Tapfer, E. Piscopiello, U. Galietti, A. Rizzo, Adhesion and wear of ZrN films sputtered on tungsten carbide substrates, Thin Solid Films, 538, 2013, 42-47.

[19] B. Tang, Y. Wang, L. Wang, X. Wang, H. Liu, Y. Yu, T. Sun, Adhesion strength of TiN films synthesized on GCr15-bearing steel using plasma immersion ion implantation and deposition, Surf. Coat. Technol., 186, 2010, 153-156.

[20] G. Cheng, S. Xu, D. Ye, Adhesion modification in $(\mathrm{Cu}, \mathrm{Nb}, \mathrm{Ti}) / \mathrm{SiO} 2$ pairs by argon ion bombardment, Nucl. Instrum. Methods:Beam Interactions with Materials and Atoms, 135(1-4), 1998, 545-549. 